\title{
Comparison between robotic and laparoscopic myomec- tomy: the introduction of a multi-center observational cohort project
}

\author{
Kuo-Chang Wen ${ }^{1,2}$, Lu-Te Chang ${ }^{1}$, Yu-Chi Wang ${ }^{3}$, Tung-Heng Lee ${ }^{4}$, Chia-Wei Chen ${ }^{1}$, Hung-Cheng Lai ${ }^{1,2,5}$ \\ ${ }^{1}$ Department of Obstetrics and Gynecology, Shuang Ho Hospital, New Taipei City; ${ }^{2}$ Department of Obstetrics and Gynecology, School of Medicine, \\ College of Medicine, Taipei Medical University, Taipei; ${ }^{3}$ Department of Obstetrics and Gynecology, Tri-Service General Hospital, National Defense \\ Medical Center, Taipei; ${ }^{4}$ Department of Obstetrics and Gynecology, Mennonite Christian Hospital, Hualien; ${ }^{5}$ Translational Epigenetic Center, Shuang \\ Ho Hospital, Taipei Medical University, New Taipei City, Taiwan
}

Objective: Uterine myomas are the most commonly encountered benign tumors of the uterus, and myomectomy may be a therapeutic option. With advancements in surgical devices, the minimally invasive approach has become the primary choice. Robot-assisted gynecological surgery is being increasingly performed; however, there is controversy regarding the advantages of robotic myomectomy (RM) compared to those of conventional laparoscopic myomectomy (LM). We design a multiple-center observational cohort project to compare the performance of RM and LM.

Methods: In this project, participants will be stratified and assigned to the RM or LM group according to the suggestion of attending physicians' and patients' choices. We will recruit 222-444 patients, aged 20-80 years old, indicated for myomectomy.

Results: The primary outcome will be the rate of conversion of RM and LM to laparotomy. The secondary outcome will include the intraoperative parameters, postoperative parameters, and duration of hospitalization. Additionally, we will attempt to generate a myoma score as the selection criteria for RM or LM.

Conclusion: This observational cohort project to compare RM and LM may provide stronger evidence regarding the advantages and disadvantages of robotic surgery for the most commonly encountered benign gynecological tumors.

Key Words: Robotic myomectomy; Laparoscopic myomectomy; Multi-center; Observational cohort

\section{INTRODUCTION}

Uterine myoma is the most common benign tumors of the uterus and may be presented $20-40 \%$ of women at reproductive age [1]. The myomas can be asymptomatic.
However, they often cause abnormal uterine bleeding, pelvic pain, compression symptom, or even infertility [1,2]. Instead of hysterectomy, myomectomy can be one option for symptomatic women who wish to preserve their childbearing capabilities $[3,4]$. Along with advances and

\footnotetext{
- Received: April 6, 2020 • Revised: September 27, 2020 •Accepted: October 7, 2020

- Correspondening author: Hung-Cheng Lai

Department of Obstetrics and Gynecology, Shuang Ho Hospital, No. 291, Zhongzheng Rd, Zhonghe District, New Taipei City 235, Taiwan

E-mail:19345@s.tmu.edu.tw
}

This is an Open Access article distributed under the terms of the Creative Commons Attribution Non-Commercial License (http://creativecommons.org/ licenses/by-nc/4.0) which permits unrestricted non-commercial use, distribution, and reproduction in any medium, provided the original work is properly cited. 
improvements in surgical devices and instruments, an increasing number of gynecologists have chosen minimally invasive procedures as the solution to treat myoma [5].

Because of small incision wound and cosmetics advantage, laparoscopic myomectomy (LM) has been confirmed in the management of uterine myoma [6,7]. However, it is very "unfriendly" for surgeons because of many disadvantages such as keeping stand for a long time, unable to relying on, or swaying visual field by first-assistant [8,9]. Since the approval by the US Food and Drug Administration (FDA) in April 2005, robotic surgeries using the da Vinci Surgical System (Intuitive Surgical Inc., Sunnyvale, CA, USA) have been applied widely in many surgical fields including urology, orthopedics, general surgery, cardiothoracic surgery, and gynecology [10]. The three-dimensional vision system and the wrist-like structure of EndoWrist instruments (Intuitive Surgical Inc.) recapitulating the motion of the surgeon's hand make precise procedures easier than in conventional laparoscopy, which allows robotic surgery to overcome some of the shortcomings and limitations of traditional laparoscopy [11]. We had also successfully undergone robotic surgery in many complicated gynecologic diseases in Taiwan [12].

A few case series comparing robotic hysterectomy with laparoscopic hysterectomy have been reported. Sarlos et al. [13] reported a randomized control trial for robotic hysterectomy and laparoscopic hysterectomy with 100 patients. Paraiso et al. [14] analyzed 62 cases of robotic hysterectomy and laparoscopic hysterectomy. Both the aforementioned groups reported that the robotic procedure was associated with longer operating times. Overall conversion to open surgery was not noted. In one case, laparoscopic hysterectomy was converted to laparotomy owing to bleeding and pneumoperitoneum failure. With respect to myomectomy, only two retrospective studies have analyzed the advantages of robotic myomectomy (RM). Bedient et al. [5] reported a chart review of 81 patients with RM; Nezhat et al. [15] reported on 15 cases of RM and 35 cases of LM. Bedient et al. [5] reported that the short-term surgical outcomes were similar between RM and LM. Conversion to an open approach was not noted in the RM group; however, two cases of conversion were reported in the LM group [5]. Nezhat et al. [15] claimed the RM required a significant prolonged surgical time over LM. They concluded that RM did not offer any substantial when per- formed by skilled laparoscopic surgeons. However, there are some limitations of these two retrospective studies. In addition to the small number of cases and non-prospective study design, the severity of each myoma assessed was not clear. The authors did not provide detailed information about the myoma location or about the cervical or broad ligament type. Selection bias is apparent and commonly noted in clinical practice. The selection of RM or conventional LM for the management of uterine leiomyomas is a dilemma for gynecologic surgeons to date. Thus, a controlled trial is needed to clarify RM's role, especially the open conversion rate.

\section{MATERIALS AND METHODS}

The authors are accountable for all work aspects in ensuring that questions related to the accuracy or integrity of any part of the work are appropriately investigated and resolved. The Institutional Review Board of TMU approved this project (N202003111).

\section{Study design}

This trial will be conducted in the hospitals of the members of the Asian Society for Gynecologic Robotic Surgery (ASGRS), and the data will be archived at Shuang Ho Hospital, Taipei Medical University, Taiwan. Fig. 1 shows the study flowchart. The primary endpoint will be the conversion rate, i.e., conversion of RM and LM to open myomectomy (OM). The secondary endpoints will be the intraoperative parameters, postoperative parameters, and duration of hospitalization. We hypothesize that lower conversion rate, less blood loss, lower re-admission rate, and shorter hospital stay duration are associated with RM than with LM, especially with respect to the treatment of treating complicated cases.

\section{Inclusion criteria}

Patients will be screened for eligibility for the trial by surgeons. Women aged $20-80$ years with symptomatic myoma will be eligible for this trial.

\section{Exclusion criteria}

Patients will be excluded if they have a history of breast cancer, gynecological cancer, or cancer treatment; if they 


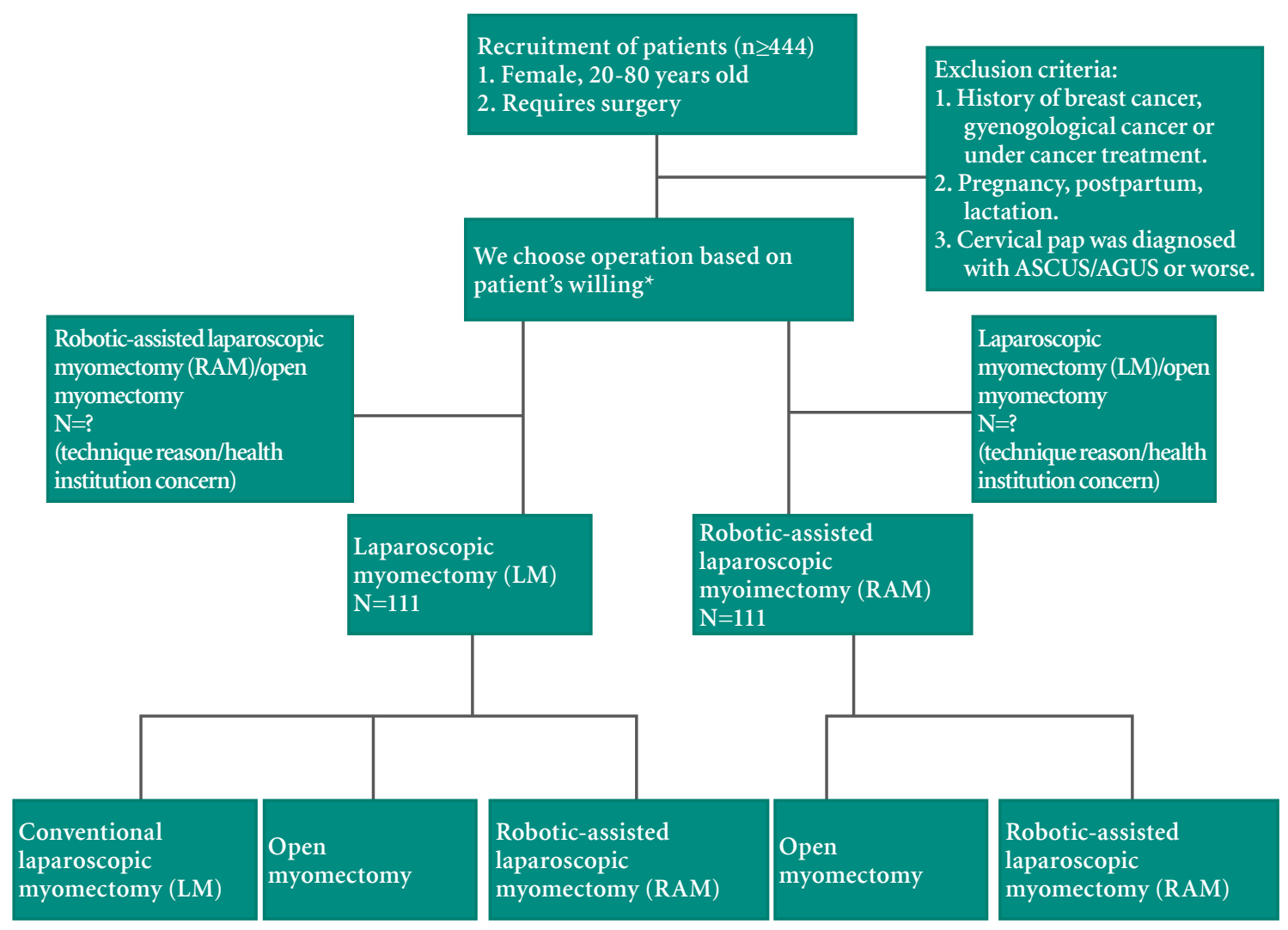

Fig. 1. Flowchart of this trial. This trial will be performed in hospitals of the Asian Society for Gynecologic Robotic Surgery members as a flowchart. The inclusion and exclusion criteria are listed. ASCUS, atypical squamous cells of undetermined significance; AGUS, atypical glandular cells of undetermined significance. *Enrolled patients choose robotic or laparoscopic myomectomy according to their willing; the Institutional Review Board of TMU also approved this project.

are pregnant, in the postpartum phase, or are breastfeeding; and if they present with abnormal Pap smear with atypical squamous cells of undertermined significance, atypical glandular cells, or worse.

\section{Participating surgeons}

We have invited members of the ASGRS to join this trial. Surgeons must be qualified for performing minimally invasive surgery in the field of gynecology. A record of performing independent laparoscopic surgeries for more than 3 years or performing robotic surgery for more than 24 patients within 1 year are minimal requirements. The case report form and related details for Institutional Review Board approval will be provided on request.

\section{Classification of uterine leiomyoma}

International Federation of Gynecology and Obstetrics (FIGO) classification of myoma will be included in our analysis. FIGO classification system for uterine myomas describes the relationship of myomas to the uterine wall [16]. The FIGO classification system retains the original submucosal relationship of types $0-2$ but extends to an additional six categories. Type 3 myoma next to the endometrium but is completely intramural. Type 4 describes an entirely intramural myoma; types 5 and 6 are defined by the relationship to the serosal layer; type 7 describes fibroids that are pedunculated on the sub-serosal surface, and type 8 refers to fibroids found in ectopic locations such as the cervix.

However, FIGO myoma classification emphasizes the site of myoma but does not precisely reflect the complexity and surgical outcome of myomectomy. We intend to generate a new classification that can reflect surgical complexity for clinical guidance. In addition to FIGO classification, the size, number, and location of myoma by ultrasound, computed tomography (CT) scan, or magnetic resonance imaging 


\begin{tabular}{|c|c|c|c|c|}
\hline Date of examination: & Ultrasound $\square /$ & MRI $\square / O r$ & ng $\square$ & \\
\hline Myoma number & FIGO type & Size $(\mathrm{cm})$ & & \\
\hline & & & $\begin{array}{l}\text { 1. Fundus } \\
\text { 2.Body } \\
\text { 3.Cervix }\end{array}$ & $\begin{array}{l}\text { 1.Anterior } \\
\text { 2.Posterior } \\
\text { 3.Lateral }\end{array}$ \\
\hline & & & & \\
\hline & & & & \\
\hline & & & & \\
\hline
\end{tabular}

Fig. 2. Myoma score form. CT, computed tomography; MRI, magnetic resonance imaging; FIGO, International Federation of Gynecology and Obstetrics.

\begin{tabular}{|c|c|c|c|c|}
\hline $\begin{array}{l}\text { Date of examination: } \\
\text { 2019/08/01 }\end{array}$ & \multicolumn{4}{|c|}{ Ultrasound $\square /$ CT $\square /$ MRI $\square$} \\
\hline \multirow[t]{2}{*}{ Myoma number } & \multirow[t]{2}{*}{ FIGO type } & \multirow[t]{2}{*}{ Size $(\mathrm{cm})$} & \multicolumn{2}{|c|}{ Location } \\
\hline & & & $\begin{array}{l}\text { 1.Fundus } \\
\text { 2.Body } \\
\text { 3.Cervix }\end{array}$ & $\begin{array}{l}\text { 1.Anterior } \\
\text { 2.Posterior } \\
\text { 3.Lateral }\end{array}$ \\
\hline$\# 1$ & 1 & $2.2 \times 1.9$ & 2 & 2 \\
\hline \#2 & 5 & $6.2 \times 5.8$ & 2 & 2 \\
\hline
\end{tabular}

Fig. 3. Myoma score for the example in Fig. 5. CT, computed tomography; MRI, magnetic resonance imaging; FIGO, International Federation of Gynecology and Obstetrics.

\begin{tabular}{|c|c|c|c|c|}
\hline \multirow{3}{*}{$\begin{array}{l}\text { Date of examination: } \\
\text { 2019/02/21 } \\
\text { Myoma number }\end{array}$} & \multicolumn{4}{|c|}{ Ultrasound $\square$ / CT $\square /$ MRI $\square$} \\
\hline & \multirow[t]{2}{*}{ FIGO type } & \multirow[t]{2}{*}{ Size $(\mathrm{cm})$} & \multicolumn{2}{|c|}{ Location } \\
\hline & & & $\begin{array}{l}\text { 1.Fundus } \\
\text { 2.Body } \\
\text { 3.Cervix }\end{array}$ & $\begin{array}{l}\text { 1.Anterior } \\
\text { 2.Posterior } \\
\text { 3.Lateral }\end{array}$ \\
\hline \#1 & 6 & $12 \times 10$ & 2 & 3 \\
\hline
\end{tabular}

Fig. 4. Myoma score for the example in Fig. 6. CT, computed tomography; MRI, magnetic resonance imaging; FIGO, International Federation of Gynecology and Obstetrics.

(MRI) will be included. The case report form of myoma is provided in Fig. 2 for the pre-operative evaluation/ operative finding of myoma. Each myoma with FIGO type, size, and location based on the sonogram, CT, or MRI, will be recorded. We distinguish the fundus, body, cervix by endometrium tip, and uterine artery insertion level, respectively. Besides, we definite anterior wall, posterior wall, and lateral area by bilateral ovary ligament. The lateral area included ovarian ligament along to the pelvic wall. Examples of myoma scoring are provided in Figs. 3-6. For example, case 1 showed multiple myomas on ultrasound
(Fig. 5). Case 2 showed a large myoma on ultrasound and CT scans (Fig. 6). The myoma information was recorded in the corresponding case report forms (Figs. 3, 4).

\section{Outcome measures}

The primary endpoint is to compare the conversion rate (transfer to OM). Patients received to LM or RM but directly undergo OM without a laparoscopy or robot try due to technical difficulties will be included in the analysis. Those who directly go for OM for insurance concerns will be excluded in the final analysis. Secondary endpoints 

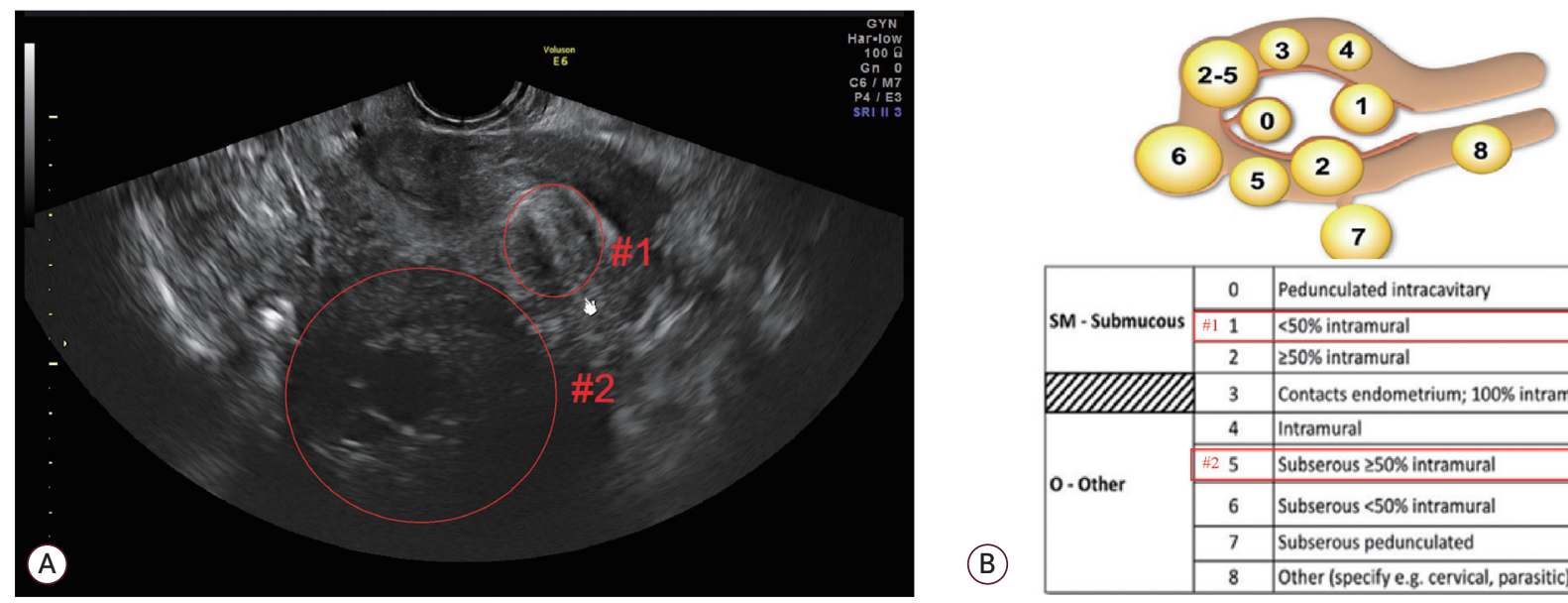

Fig. 5. Sonography of uterine myoma and FIGO classification system. The case showed multiple myomas by ultrasound: (A) FIGO myoma calcification [16], (B) type 1 (\#1) and type 5 (\# 2), respectively. FIGO, International Federation of Gynecology and Obstetrics.
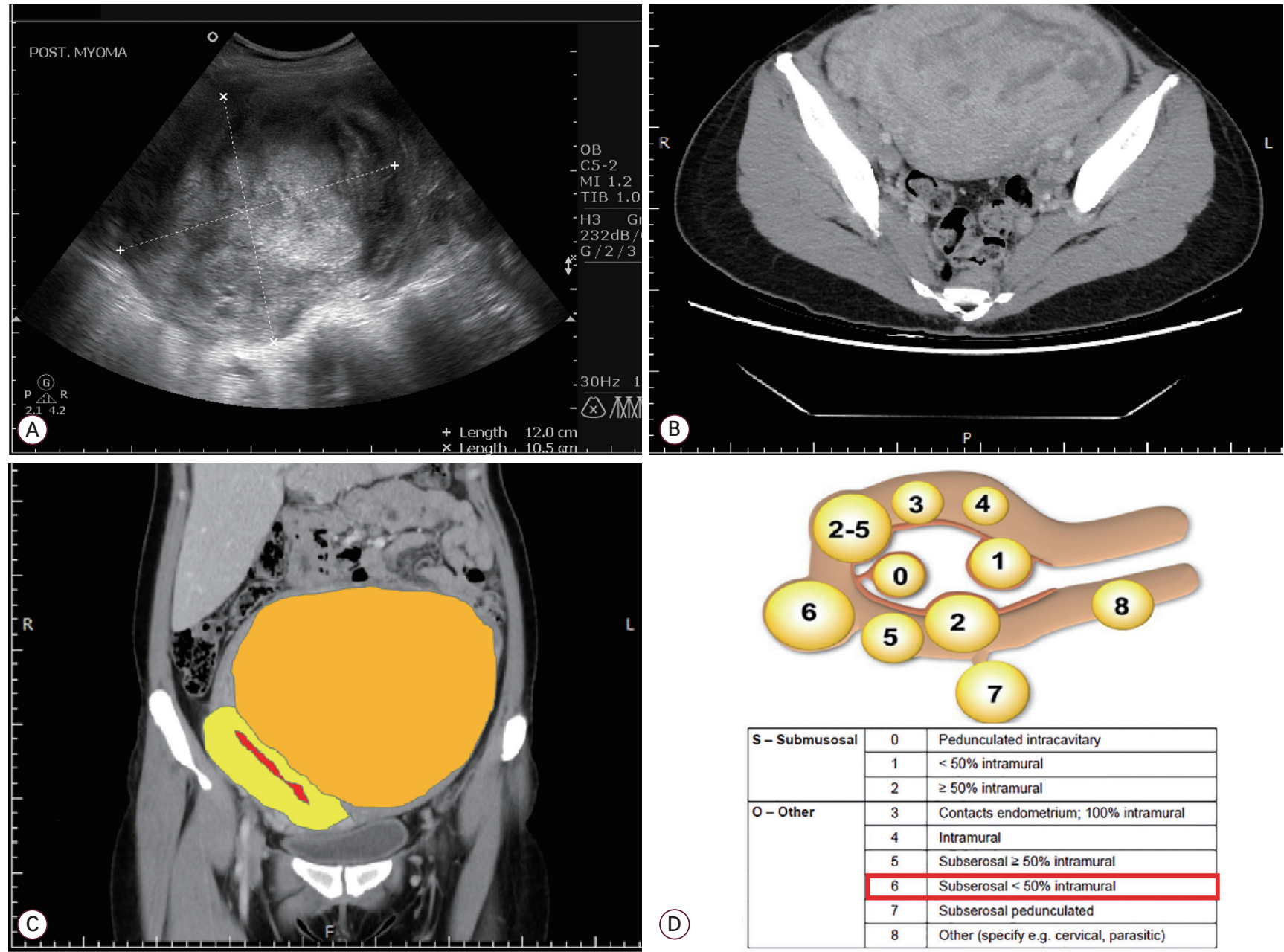

7

\begin{tabular}{|l|c|l|}
\hline S - Submusosal & 0 & Pedunculated intracavitary \\
\cline { 2 - 3 } & 1 & $<50 \%$ intramural \\
\cline { 2 - 3 } & 2 & $\geq 50 \%$ intramural \\
\hline \multirow{5}{*}{ O- Other } & 3 & Contacts endometrium; $100 \%$ intramural \\
\cline { 2 - 3 } & 4 & Intramural \\
\cline { 2 - 3 } & 5 & Subserosal $\geq 50 \%$ intramural \\
\cline { 2 - 3 } & 6 & Subserosal $<50 \%$ intramural \\
\cline { 2 - 3 } & 7 & Subserosal pedunculated \\
\cline { 2 - 3 } & 8 & Other (specify e.g. cervical, parasitic) \\
\hline
\end{tabular}

Fig. 6. Computed tomography (CT) scan of uterine myoma and FIGO calcification. The case showed one huge myoma by ultrasound (A) and CT scan (B, C); FIGO myoma calcification [16] presented type 6 (D). FIGO, International Federation of Gynecology and Obstetrics. 
include treatment-related morbidity as evaluated by the incidence of: 1) intraoperative complications (injury to the bladder, ureters, bowel, blood vessels, and bleeding), 2) estimated blood loss and rate of blood transfusion, 3) re-admission within one month, and 4) days of hospitalization.

We also assess clinical parameters such as operation time, docking time, and console time. First, operation time is calculated from skin incision to skin closure. Docking time is then calculated from skin incision to all trocars connected with robotic arms. Lastly, console time is defined as the time from the surgeon finishing docking to finishing the robotic console performance.

Because there was no myoma score associated with the difficulty of operation, we generated a new myoma classification score predicting the risks of morbidities, including conversion rate, for future patient selection guidance. We also identified the quality factors of operation, such as operation time and blood loss, and includes the myoma characteristics such as the number, size, type, location, and FIGO type to create a multivariable myoma score model linear regression. When the surgeons key in the individual case's myoma characteristic, the clinical outcomes will be predicted by the new myoma score model. The score is proportional to the operation's difficulty and index to evaluate the appropriate approach for myomectomy.

\section{Statistics considerations}

\section{1) Prospective observational study}

Due to the limitations of national health insurance, it is not easy to randomize the participants to RM or LM. We will check the patient's willingness to undergo minimally invasive surgery. If patients tend to undergo minimally invasive surgery, they cannot afford RM. They will be assigned to the LM group, even though the surgeon suggests $O M$. These cases will be documented and analyzed with the reference rate/distribution rate. Subjects will be labeled with a serial number (and therefore the name, name abbreviations, chart number, personal identification number, telephone number, fax number, mail address, and other information that lead to personal identification will not appear). The results will be stored and shared by all principal investigators.

\section{2) Sample size estimation}

In this trial, we aim to assess the difference of laparoscopic conversion rate between robotic surgery $\left(P_{\text {(Robotic) }}=1 \%\right)$ and laparoscopic surgery $\left(\mathrm{P}_{\text {(Laparoscoov) }}=10 \%\right)$. In our experience, we performed over 100 cases of RM in past years, and there was no case to convert to OM. Since we imported the robotic platform in 2014, we found that the percentage of OM decreased, and RM increased gradually (Fig. 7). However, the percentage of LM was similar. We supposed that some cases with complicated myoma were referred to RM, which is difficult for the laparoscopic approach. Besides, there was no randomized control trial in this issue, and Gobern et al. [17] reported a conversion rate of LM is $8 \%$. Thus, we presume that the conversion rate of robotic surgery is $1 \%$ and $10 \%$ in laparoscopic surgery. This sample size is based on a power of $80 \%$ and a two-tailed $P<0.05$ as significant. Also, we assumed that $5 \%$ of cases will lose to follow-up. Finally, case numbers in each group of robotic surgery and laparoscopic surgery are 111, and the total sample size is 222 .

\section{Analysis}

The 444 cases' data were analyzed along with both the conversion rate and both group's characteristics. All statistical analyses and debugging will use IBM SPSS Statistics for Windows, version 22.0 (IBM Corp., Armonk, NY, USA). Continuous variables are presented as median and interquartile, and categorical variables are presented as num-

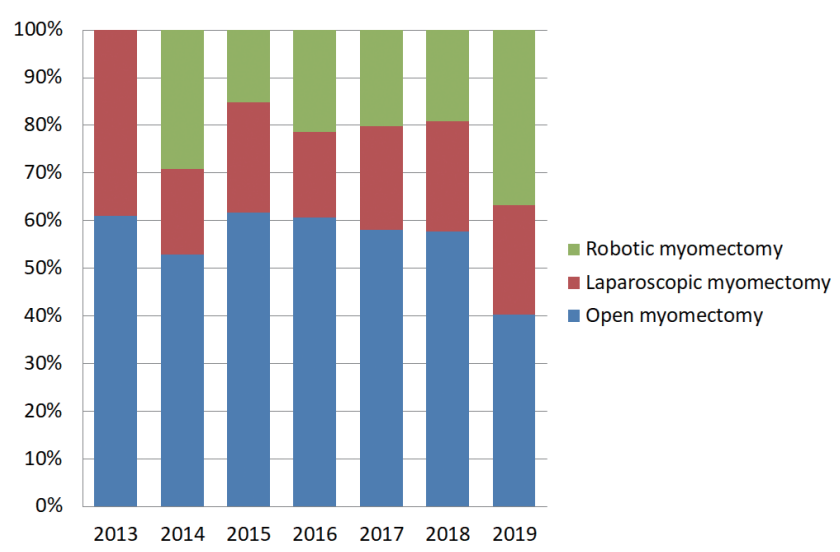

Fig. 7. The trend of myomectomy. The percentage of robotic myomectomy procedures performed gradually increased after we imported the robotic platform between 2013 till 2019 in Shuang Ho Hospital. 
bers (n) and percentages (\%). The two-sample t-test (nonparametric Mann-Whitney $U$ test) will be used to identify the difference in mean and standard deviation (medians and variants) between the two groups, such as age, body mass index, the characteristics of myomas, operation time, ducking time, console time, blood loss, and days of hospitalization. The chi-square test will identify the difference in the laparotomy conversion rate between the two groups. All measures will be compared by intention-to-treat analysis, including all randomized patients. The $95 \%$ confidence interval will be reported for the differences between the treatment arms.

\section{RESULTS}

We introduce the study design and flowchart to compare robotic and laparoscopic myomectomy. This observation cohort project is on the way and the results will be analyzed in future.

\section{DISCUSSION}

Since the FDA approved the robotic platform in the gynecological field in 2005, it has been a mature surgical technique for gynecological diseases. There is still an issue of learning curves in robotic surgery, although the platform is more user-friendly than laparoscopic surgery. Woelk et al. [18] found that operative time decreased significantly from 3.5 to 2.7 hours during 3 years of robotic hysterectomy. The proportion of patients with a stay longer than 1 day significantly decreased from $49.2 \%$ to $14.7 \%$. Chen et al. [19] reported a steep decrease in docking time and console time in the robotic single-site hysterectomy group given chronological change. Although no study has addressed RM's learning curve, we assume it is similar to robotic hysterectomy [20]. Therefore, we assumed 24 cases within 1 year could master the technique needed for RM.

Myoma is heterogeneous in size, location, and FIGO type. These characteristics consist of complexity and directly influence the operation outcome. Thus, myomectomy surgery is individualized and difficult to standardize [21]. There is no score yet that is clinically helpful for risk stratification of minimally invasive myomectomy. Thus, we try to develop a risk stratification score, like the prediction of the adherent placenta in placenta previa [22], which will help the patient selection of RM, LM, or OM.

The initial trial was proposed for the collaborative effort of the ASGR members. Through this cooperation, ASGRS will strengthen the network and contribute to women's health.

\section{Conflict of interest}

No potential conflict of interest relevant to this article was reported.

\section{Acknowledgments}

This work was supported by the Ministry of Science and Technology, Executive Yuan, Taiwan (MOST 109-2314-B038-055- to KCW), Shuang Ho Hospital, Taipei Medical University (TMU108-AE1-B54 to KCW), and Teh-Tzer Study Group for Human Medical Research Foundation.

\section{References}

1. Practice Committee of American Society for Reproductive Medicine in collaboration with Society of Reproductive Surgeons. Myomas and reproductive function. Fertil Steril 2008;90(5 Suppl):S125-30.

2. Shwayder J, Sakhel K. Imaging for uterine myomas and adenomyosis. J Minim Invasive Gynecol 2014;21:362-76.

3. Cheng $\mathrm{MH}$, Chao HT, Wang PH. Medical treatment for uterine myomas. Taiwan J Obstet Gynecol 2008;47:18-23.

4. Cheng $\mathrm{MH}$, Wang $\mathrm{PH}$. Uterine myoma: a condition amenable to medical therapy? Expert Opin Emerg Drugs 2008;13:119-33.

5. Bedient CE, Magrina JF, Noble BN, Kho RM. Comparison of robotic and laparoscopic myomectomy. Am J Obstet Gynecol 2009;201:566.e1-5.

6. Palomba S, Zupi E, Falbo A, Russo T, Marconi D, Tolino A, et al. A multicenter randomized, controlled study comparing laparoscopic versus minilaparotomic myomectomy: reproductive outcomes. Fertil Steril 2007;88:933-41.

7. Palomba S, Zupi E, Russo T, Falbo A, Marconi D, Tolino A, et al. A multicenter randomized, controlled study comparing laparoscopic versus minilaparotomic myomectomy: short-term outcomes. Fertil Steril 2007;88:942-51.

8. Alessandri F, Lijoi D, Mistrangelo E, Ferrero S, Ragni N. Randomized study of laparoscopic versus minilaparotomic myomectomy for uterine myomas. J Minim Invasive Gynecol 2006;13:92-7.

9. Jin C, Hu Y, Chen XC, Zheng FY, Lin F, Zhou K, et al. Laparoscopic 
versus open myomectomy--a meta-analysis of randomized controlled trials. Eur J Obstet Gynecol Reprod Biol 2009;145:14-21.

10. Advincula AP, Wang K. Evolving role and current state of robotics in minimally invasive gynecologic surgery. J Minim Invasive Gynecol 2009;16:291-301.

11. Nezhat C, Lavie O, Lemyre M, Unal E, Nezhat CH, Nezhat F. Robot-assisted laparoscopic surgery in gynecology: scientific dream or reality? Fertil Steril 2009;91:2620-2.

12. Tan SJ, Lin CK, Fu PT, Liu YL, Sun CC, Chang CC, et al. Robotic surgery in complicated gynecologic diseases: experience of tri-service general hospital in Taiwan. Taiwan J Obstet Gynecol 2012;51:1825.

13. Sarlos D, Kots L, Stevanovic N, von Felten S, Schär G. Robotic compared with conventional laparoscopic hysterectomy: a randomized controlled trial. Obstet Gynecol 2012;120:604-11.

14. Paraiso MF, Ridgeway B, Park AJ, Jelovsek JE, Barber MD, Falcone $\mathrm{T}$, et al. A randomized trial comparing conventional and robotically assisted total laparoscopic hysterectomy. Am J Obstet Gynecol 2013;208:368.e1-7.

15. Nezhat C, Lavie O, Hsu S, Watson J, Barnett O, Lemyre M. Robotic-assisted laparoscopic myomectomy compared with standard laparoscopic myomectomy--a retrospective matched control study. Fertil Steril 2009;91:556-9.

16. Munro MG, Critchley HO, Broder MS, Fraser IS; FIGO Work- ing Group on Menstrual Disorders. FIGO classification system (PALM-COEIN) for causes of abnormal uterine bleeding in nongravid women of reproductive age. Int J Gynaecol Obstet 2011;113:3-13.

17. Gobern JM, Rosemeyer CJ, Barter JF, Steren AJ. Comparison of robotic, laparoscopic, and abdominal myomectomy in a community hospital. JSLS 2013;17:116-20.

18. Woelk JL, Casiano ER, Weaver AL, Gostout BS, Trabuco EC, Gebhart JB. The learning curve of robotic hysterectomy. Obstet Gynecol 2013;121:87-95.

19. Chen CW, Chang HC, Huang TF, Liao CC, Huang RL, Lai HC. Transition from multiport to single-site surgery: a single institution experience in robotic supracervical hysterectomy for benign gynecological diseases. Taiwan J Obstet Gynecol 2019;58:514-9.

20. Varghese A, Doglioli M, Fader AN. Updates and controversies of robotic-assisted surgery in gynecologic surgery. Clin Obstet Gynecol 2019;62:733-48.

21. Rakotomahenina H, Rajaonarison J, Wong L, Brun JL. Myomectomy: technique and current indications. Minerva Ginecol 2017;69:357-69.

22. Tanimura K, Morizane M, Deguchi M, Ebina Y, Tanaka U, Ueno Y, et al. A novel scoring system for predicting adherent placenta in women with placenta previa. Placenta 2018;64:27-33. 